\title{
The role of arachidonic acid in a rat renal ischemia-reperfusion injury model
}

\author{
MASAHIDE MATSUYAMA ${ }^{1}$, RIKIO YOSHIMURA ${ }^{1}$, KIYOAKI FUNAO ${ }^{1}$, YUTAKA KAWAHITO ${ }^{2}$, \\ HAJIME SANO ${ }^{3}$, JAMEL CHARGUI ${ }^{4}$, JEAN-LOUIS TOURAINE ${ }^{4}$ and TATSUYA NAKATANI ${ }^{1}$ \\ ${ }^{1}$ Department of Urology, Osaka City University Graduate School of Medicine, 1-4-3 Asahi-machi, \\ Abeno-ku, Osaka 545-8585; ${ }^{2}$ Department of Inflammation and Immunology, Graduate School of Medical Science, \\ Kyoto Prefectural University of Medicine, 465 Kajiicho, Kawara-machi, Kamigyou-ku, Kyoto 602-0841; \\ ${ }^{3}$ Department of Internal Medicine, Hyogo College of Medicine, 1-1 Mukogawa-cho, Nishinomiya, Hyogo 663-8501, \\ Japan; ${ }^{4}$ Department of Transplantation and Clinical Immunology, Claude Bernard University of Lyon \\ and Lyon Hospitals, Pavillion P, Hôpital Edouard Herriot, 69437 Lyon Cedex 3, France
}

Received February 19, 2008; Accepted April 4, 2008

\begin{abstract}
The metabolism of arachidonic acid by either the cyclooxygenase (COX) or the lipoxygenase (LOX) pathway generates eicosanoids, which have been implicated in the pathogenesis of a variety of human diseases, including ischemia-reperfusion (I/R) injury. Several reports have demonstrated that COX-2 and LOX inhibitors can reduce the damage caused by I/R injury. However, few reports have investigated the effects of COX and LOX expression on renal I/R injury, thus this study aimed to do so in a rat renal I/R injury model. The right kidney was harvested and the left renal artery and vein were clamped under laparotomy. The kidney was reperfused after $90 \mathrm{~min}$ of ischemia, and rats were sacrificed at 0 , $1.5,3,5,12$ and $24 \mathrm{~h}$ after reperfusion. COX and LOX expression was analyzed by immunohistochemistry. COX-2 and 5- and 12-LOX expression was most intense in the endothelial cells at 3 and $5 \mathrm{~h}$ after reperfusion. The expression of COX-2 was stronger than that of 5- and 12-LOX. However, in the hours following reperfusion there were no significant variations in COX-1 expression. Our results demonstrate that COX-2 and LOX can be induced in a rat renal I/R injury model, and that the arachidonic acid pathways play a very important role in renal I/R injury.
\end{abstract}

\section{Introduction}

Renal transplantation is an acceptable therapeutic approach for patients with end-stage renal disease. However, renal ischemia-reperfusion (I/R) injury, a clinically significant prob-

Correspondence to: Dr Rikio Yoshimura, Department of Urology, Osaka City University Hospital, 1-4-3 Asahi-machi, Abeno-ku, Osaka 545-8585, Japan

E-mail: jasmin@med.osaka-cu.ac.jp

Key words: cyclooxygenase-2, lipoxygenases, renal ischemiareperfusion injury, acute tubular necrosis lem and the invariable consequence of renal transplantation, may result from aortic cross-clamping and resusciation after systemic hypotension. Recent I/R injury studies have focused on the function of neutrophils, the action mechanisms of inflammatory cytokines, tissue factor, intercellular adhesion molecule-1, oxygen free radicals, vascular plugging, edema and other complications (1).

In the process of $\mathrm{I} / \mathrm{R}$ injury, inflammatory reactions are activated and inflammatory cytokines, such as tumor necrosis factor- $\alpha$ and interleukin-1ß, or arachidonic acid metabolites, such as thromboxane and prostaglandin $\mathrm{I}_{2}$, prostaglandin $\mathrm{E}_{2}$ or leukotrienes, are released (2). The metabolisation of arachidonic acid by either the cyclooxygenase (COX) or the lipoxygenase (LOX) pathway generates eicosanoids, which have been implicated in the pathogenesis of a variety of human diseases. Arachidonic acid is metabolized through pathways involving 5- and 12-LOX, which catayzes the conversion of the acid to leukotrienes, and COX-1 and -2 , which catalyzes its converstion to thromboxanes and prostaglandins, producing a host of pro-inflammatory eicosanoids (3-5).

COX-1 is constitutively expressed in most tissues, whereas COX-2 is induced in response to pro-inflamamatory cytokines and stress. Investigators have also shown that COX-2 plays an important role in basal organ and tissue homeostasis (3). 5- and 12-LOX convert arachidonic, linoleic and other polyunsaturated fatty acids into biologically active metabolites that influence cell signaling, structure and metabolism (6).

Recently, several reports suggested that the administration of COX-2 and LOX inhibitors could alleviate I/R injury. However, few reports have addressed the connection between renal I/R injury and changes in arachidonic acid pathway expression. In this study, we examined these changes in a rat renal I/R injury model in the hours following renal I/R injury.

\section{Materials and methods}

Ischemia-reperfusion model. Male Lewis rats (180-230 g) were used in this study. Under laparotomy using pentobarbital sodium anesthesia, the right kidney was harvested and the left 

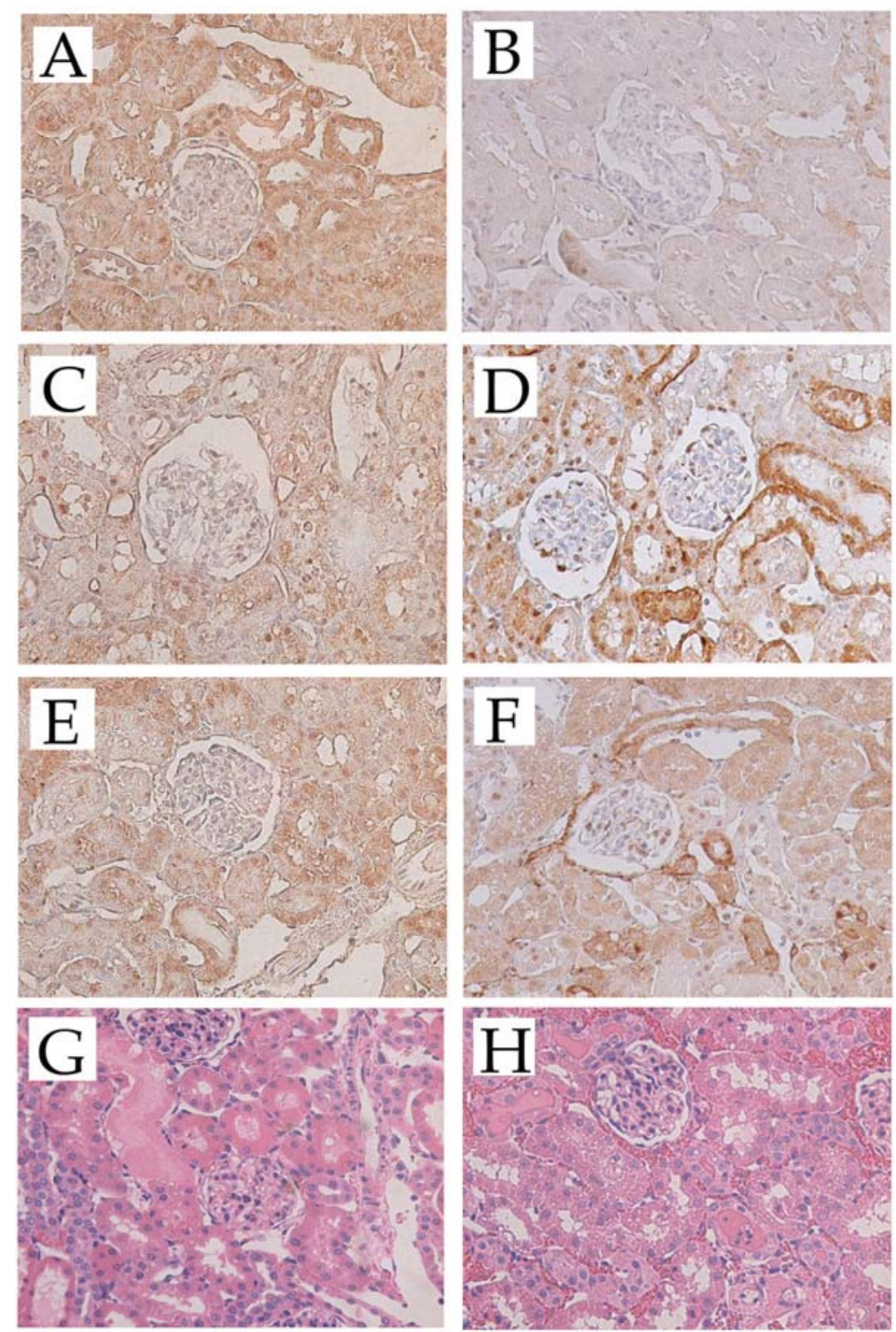

Figure 1. COX-1 and -2 immunohistochemistry and H\&E staining. A, COX-1 expression in a normal kidney; B, COX-2 expression in a normal kidney; C, COX-1 expression at $3 \mathrm{~h}$ after reperfusion; D, COX-2 expression at $3 \mathrm{~h}$ after reperfusion; E, COX-1 expression at $12 \mathrm{~h}$ after reperfusion; F, COX-2 expression at $12 \mathrm{~h}$ after reperfusion; G, H\&E staining at $5 \mathrm{~h}$ after reperfusion; $\mathrm{H}, \mathrm{H} \& \mathrm{E}$ staining at $12 \mathrm{~h}$ after reperfusion. There were no variations in COX-1 expression in the hours following reperfusion (A, C and E). COX-2 expression was only observed in the endothelial cells of normal kidneys (B). COX-2 expression was most intense in the endothelial cells at $3 \mathrm{~h}$ after reperfusion (D), but became weaker at $12 \mathrm{~h}$ after reperfusion (F). Five hours after reperfusion, the internal spaces of the tubular epithelial cells were expanded and slight destruction was apparent (G). Twelve hours after reperfusion, necrosis extended throughout the ischemic kidney and nearly all the tubular epithelial cells were destroyed $(\mathrm{H})$.

renal artery and vein were clamped with a hemostasis clip for $90 \mathrm{~min}$. The clip was subsequently removed to permit reperfusion, and the abdomen was closed during $\mathrm{I} / \mathrm{R}$. The rats were sacrificed at $0,1.5,3,5,12$ and $24 \mathrm{~h}$ after reperfusion, and their kidneys harvested. Samples of ischemic and non-ischemic kidney tissue were fixed in $10 \%$ buffered formalin for $24 \mathrm{~h}$ for H\&E staining and immunohistochemistry.

Immunohistochemistry. Immunohistochemistry was performed with the Vectastatin avidin-biotin peroxidase complex kit (Vector Laboratories, Burlingame, CA, USA). Kidney tissues sectioned onto microscope slides were deparaffinized, then the slides were immersed for $45 \mathrm{~min}$ in $0.3 \%$ peroxide in methanol to deplete endogenous peroxidase activity. Non-specific binding sites were saturated with $0.2 \%$ bovine serum albumin and normal goat serum diluted 1:66.7 in PBS for 20 min. Primary antibodies against rabbit COX-1 and -2 (Vector Laboratories), rabbit 5-LOX (Cayman Chem, USA) and 12-LOX (Oxford Biomedical Research, USA) and control normal rabbit serum (Vector Laboratories) at a dilution of 1:100 were applied to tissue sections, which were then incubated in a humidified chamber at room temperature for $30 \mathrm{~min}$, then washed with PBS for $10 \mathrm{~min}$. Biotinylated goat anti-rabbit IgG (Vector Laboratories) was applied to the tissue sections, which were incubated at room temperature for $30 \mathrm{~min}$. After being washed with PBS for $10 \mathrm{~min}$, the slides were incubated with avidinbiotinylated peroxidase (Vector Laboratories) for $45 \mathrm{~min}$. Finally, color was developed by the immersion of the sections in a peroxidase substrate solution including $0.02 \%$ peroxide, 3,3'diaminobenzine tetrahydrochloride, $0.04 \%$ nickel chloride and $0.01 \%$ hydrogen peroxide in $0.05 \mathrm{M}$ Tris- $\mathrm{HCl}, \mathrm{pH} 7.2$, for 2-7 $\mathrm{min}$. 

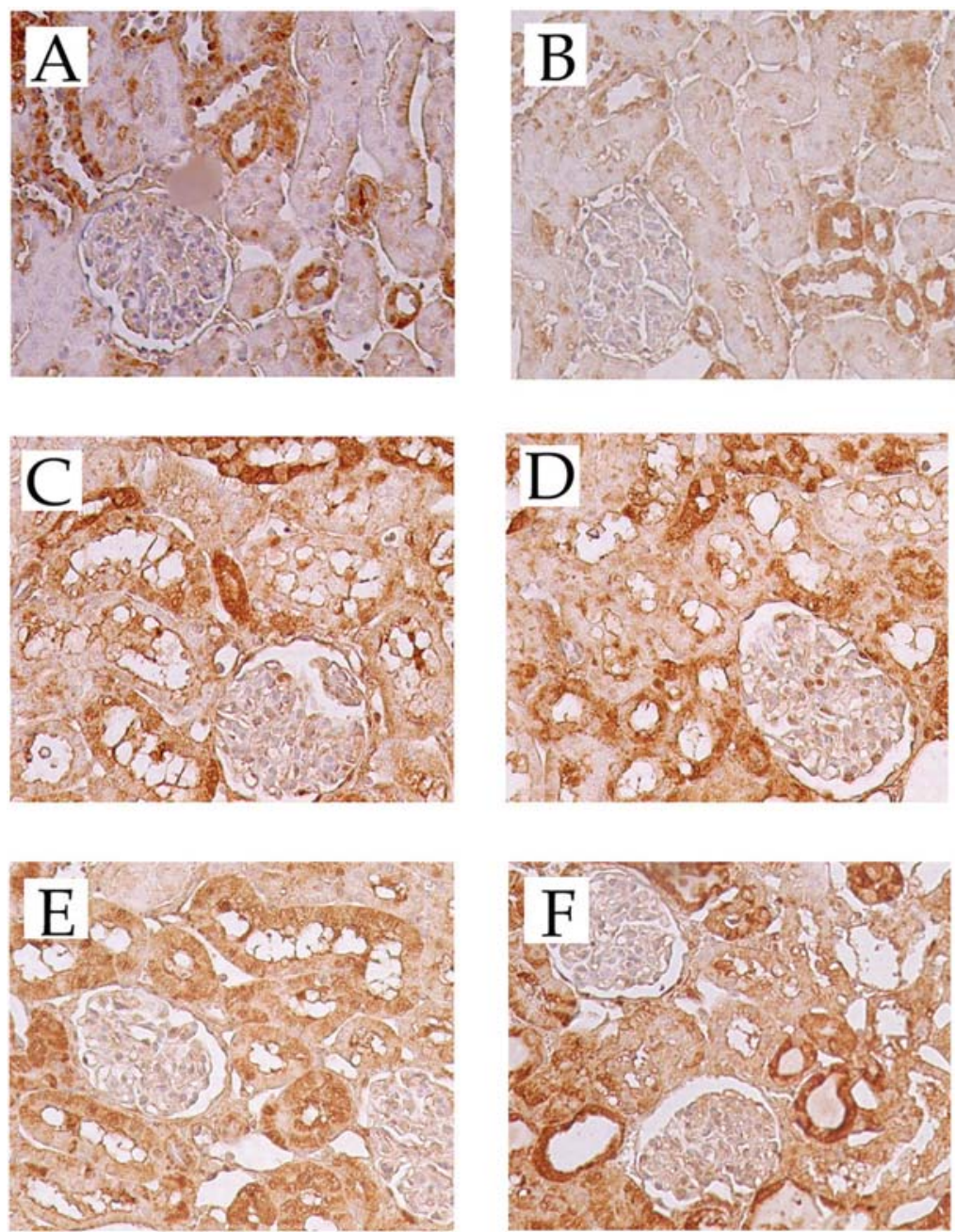

Figure 2. 5- and 12-LOX immunohistochemistry. A, 5-LOX expression in a normal kidney; B, 12-LOX expression in a normal kidney; C, 5-LOX expression at $3 \mathrm{~h}$ after reperfusion; D, 12-LOX expression at $3 \mathrm{~h}$ after reperfusion; E, 5-LOX expression at $12 \mathrm{~h}$ after reperfusion; F, 12-LOX expression at $12 \mathrm{~h}$ after reperfusion. 5- and 12-LOX expression was only observed in the endothelial cells of normal kidneys (A and B). 5- and 12-LOX expression was most intense in the endothelial cells at $3 \mathrm{~h}$ after reperfusion $(\mathrm{C}$ and $\mathrm{D})$, but became weaker at $12 \mathrm{~h}$ after reperfusion $(\mathrm{E}$ and $\mathrm{F})$.

Analysis of acute tubular necrosis, COX-1 and -2 expression score. To quantify the degree of acute tubular necrosis (ATN), the ATN was graded on scale of 0-3 by two observers on two separate occasions in a blinded manner using coded slides, then an average score was evaluated and samples were defined as having no, mild, moderate or severe necrosis. Necrosis, capillary congestion, interstitial edema, cast, destroyed, flat and extended areas of tubular epithelial cells were also evaluated. Similarly, COX and LOX immunostainings were graded on a scale of 0-4 according to the intensity of immunostaining, with four grades being the maximum. To quantify ATN, COX and LOX expression, the same two pathologists performed assessments throughout the study.

Statistical analysis. All results are presented as the means \pm $\mathrm{SD}$. Analysis of the data was performed using the analysis of variance (ANOVA) (7).

\section{Results}

$H \& E$ staining and COX and LOX immunohistochemistry. COX-2, 5- and 12-LOX expression was only observed in the endothelial cells of normal kidneys (Figs. 1B, 2A and B).
From 1.5 to $3 \mathrm{~h}$ after reperfusion, COX-2, 5- and 12-LOX expression became gradually stronger in the endothelial cells, reaching peak intensity at 3 and $5 \mathrm{~h}$ after reperfusion (Figs. 1D, $2 \mathrm{C}$ and $\mathrm{D})$. COX-2 expression was stronger than 5- and 12-LOX expression.

Five hours after reperfusion, the internal spaces of the tubular epithelial cells had expanded and slight destruction was apparant (Fig. 1G). Twelve hours after reperfusion, necrosis had extended throughout the ischemic kidney and nearly all the tubular epithelial cells were destroyed (Fig. 1H). From 12 to $24 \mathrm{~h}$ after reperfusion, COX-2, 5-and 12-LOX expression gradually became weaker in the endothelial cells (Figs. 1F, 2E and $\mathrm{F}$ ). There were no variations in $\mathrm{COX}-1$ expression in the hours following reperfusion (Fig. 1A, C and E).

Statistical analysis of COX and LOX expression and acute tubular necrosis score. ATN scores (Table I) gradually increased with the passage of time after reperfusion $(0 \mathrm{~h}$, $0.1 \pm 0.2 ; 1.5 \mathrm{~h}, 0.2 \pm 0.3 ; 3 \mathrm{~h}, 0.6 \pm 0.4 ; 5 \mathrm{~h}, 1.2 \pm 0.4 ; 12 \mathrm{~h}$, $2.5 \pm 0.4 ; 24 \mathrm{~h}, 2.9 \pm 0.3)$. COX-1 expression scores (Table I) did not differ significantly from hour to hour $(0 \mathrm{~h}, 1.2 \pm 0.7 ; 1.5 \mathrm{~h}$, $1.4 \pm 0.7 ; 3 \mathrm{~h}, 1.5 \pm 0.8 ; 5 \mathrm{~h}, 1.6 \pm 0.8 ; 12 \mathrm{~h}, 1.8 \pm 0.8 ; 24 \mathrm{~h}$, $1.4 \pm 0.6$; before ischemia, $1.1 \pm 0.6)$. However, COX-2 expres- 
Table I. Statistical analysis of COX and LOX expression and of ATN score.

\begin{tabular}{cccccc}
\hline & \multicolumn{4}{c}{ Mean \pm SD } \\
\cline { 2 - 5 } Time (h) after I/R injury & COX-1 & COX-2 & 5 -LOX & 12-LOX & ATN \\
\hline 0 & $1.2 \pm 0.7$ & $0.8 \pm 0.4$ & $1.7 \pm 0.7$ & $0.9 \pm 0.6$ & $0.1 \pm 0.2$ \\
1.5 & $1.4 \pm 0.7$ & $1.9 \pm 0.6^{\mathrm{a}}$ & $2.0 \pm 0.7$ & $1.7 \pm 0.8$ & $0.2 \pm 0.3$ \\
3 & $1.5 \pm 0.8$ & $3.1 \pm 0.5^{\mathrm{a}}$ & $2.3 \pm 0.8$ & $2.4 \pm 0.9^{\mathrm{a}}$ & $0.6 \pm 0.4$ \\
5 & $1.6 \pm 0.8$ & $3.3 \pm 0.6^{\mathrm{a}}$ & $2.4 \pm 0.8$ & $2.7 \pm 0.6^{\mathrm{a}}$ & $1.2 \pm 0.4$ \\
12 & $1.8 \pm 0.8$ & $2.5 \pm 0.7^{\mathrm{a}}$ & $1.8 \pm 0.8$ & $1.9 \pm 0.7^{\mathrm{a}}$ & $2.5 \pm 0.4$ \\
24 & $1.4 \pm 0.6$ & $1.8 \pm 0.7$ & $1.7 \pm 0.8$ & $1.6 \pm 0.7$ & $2.9 \pm 0.3$ \\
Before ischemia & $1.1 \pm 0.6$ & $0.6 \pm 0.5$ & $1.2 \pm 0.7$ & $0.8 \pm 0.4$ & $\mathrm{NP}$ \\
\hline
\end{tabular}

COX and LOX immunostainings were assigned a grade of 0-4 and ATN a grade of 0-3 by two observers in a blinded manner. Statistical analysis was performed using the analysis of variance (p-value; ANOVA). ${ }^{\mathrm{a}} \mathrm{p}<0.01$.

sion scores were significantly higher at $1.5,3,5,12$ and $24 \mathrm{~h}$ after reperfusion as compared to $0 \mathrm{~h}$ after reperfusion $(0 \mathrm{~h}$, $0.8 \pm 0.4 ; 1.5 \mathrm{~h}, 1.9 \pm 0.6 ; 3 \mathrm{~h}, 3.1 \pm 0.5 ; 5 \mathrm{~h}, 3.1 \pm 0.6 ; 12 \mathrm{~h}$, $2.5 \pm 0.7 ; 24$ h, $1.8 \pm 0.7$; before ischemia, $0.6 \pm 0.5$ ).

5- and 12-LOX expression scores are shown in Table I. The 12-LOX expression score $(0 \mathrm{~h}, 0.9 \pm 0.6 ; 1.5 \mathrm{~h}, 1.7 \pm 0.8$; $3 \mathrm{~h}, 2.4 \pm 0.9 ; 5 \mathrm{~h}, 2.7 \pm 1.0 ; 12 \mathrm{~h}, 1.9 \pm 0.7 ; 24 \mathrm{~h}, 1.6 \pm 0.7$; before ischemia, $0.8 \pm 0.4)$ was more inducible than the 5 -LOX expression score $(0 \mathrm{~h}, 1.7 \pm 0.7 ; 1.5 \mathrm{~h}, 2.0 \pm 0.7 ; 3 \mathrm{~h}, 2.3 \pm 0.8$; $5 \mathrm{~h}, 2.4 \pm 0.8 ; 12 \mathrm{~h}, 1.8 \pm 0.8 ; 24 \mathrm{~h}, 1.7 \pm 0.8$; before ischemia, $1.2 \pm 0.7$ ) in renal $\mathrm{I} / \mathrm{R}$ injury.

\section{Discussion}

Renal injury after I/R occurs as a consequence of tissue hypoxia from interrupted blood supply or from the process of reperfusion that leads to an active inflammatory process. Infiltrating leukocytes are a potential source of reactive oxygen, proteolytic enzymes or cytokines, and may play a detrimental role during reperfusion (1).

COX, which produces prostanoids from arachidonic acid, is the principal target of non-steroidal anti-inflammatory drugs. Recently, two types of COX isoform (COX-1 and -2) were identified. COX-1 is present in most tissues and is involved in the physiological production of prostaglandins, which are involved in the maintentance and regulation of normal homeostatic function. COX-2, on the other hand, has been well established to participate in prostanoid production and is implicated in the disease process. It is expressed in inflammatory sites, which often have harmful effects and are associated with pain, fever, lipopolysaccharide stimulation and I/R injury (8).

In this study, we demonstrated that COX-2, 5- and 12-LOX were expressed in a rat renal I/R injury model. From 3 to $5 \mathrm{~h}$ after I/R injury, COX-2, 5- and 12-LOX expression was most intense. Maximum tissue damage was observed 12-24 h after $\mathrm{I} / \mathrm{R}$ injury. However, there were no variations in COX-1 expression in the hours following reperfusion. Several reports support the notion that COX-1 mRNA is not affected by $I / R$ $(9,10)$. In this study in particular, COX-2 and 12-LOX expres- sion was more inducible than that of 5-LOX. In seeming support of our data, Takamatsu et al reported that leukotriene mRNA, namely 5-LOX, is not affected by hepatic I/R (11).

Generally, it is possible to demonstrate that the arteriolar extension of prostaglandin $\mathrm{I}_{2}$ inhibition of platelet aggregation, the induction of thromboxane $\mathrm{A}_{2}$ by platelet aggregation, vasoconstriction and bronchoconstriction are directly affected by prostaglandin. It is thought that thromboxane $\mathrm{A}_{2}$ antagonizes prostaglandin $\mathrm{I}_{2}$ and is involved in $\mathrm{I} / \mathrm{R}$ injury. Takeyoshi et al reported that peroral administration of FK3311 (1 mg/kg) COX-2 inhibitor reduced the degree of neutrophilic leukocyte invasion, tissue destruction and thromboxane $\mathrm{B}_{2}$ at $6 \mathrm{~h}$ after reperfusion in a $60 \mathrm{~min}$ dog renal warm $\mathrm{I} / \mathrm{R}$ injury model (12), and that with the administration of this drug, the 2-day survival rate was significantly better than in the control group in a dog 15 min lung warm I/R injury model (13).

LOX occurs in various isoforms (5-, 8-, 12- and 15-LOX). 5 -LOX is an enzyme induced by cytokines that synthesizes various leukotrienes from arachidonic acid. The leukotriene $\mathrm{A}_{4}$ has poor biological activity, while the leukotriene $\mathrm{B}_{4}$ affects neutrophilic leukocyte extravascular migration and venula constriction. The leukotrienes $\mathrm{C}_{4}$, leukotriene $\mathrm{D}_{4}$ and leukotriene $\mathrm{E}_{4}$ affect plain muscle contraction, blood vessel permeability, and thromboxane $\mathrm{A}_{2}$ and the accentuation of platelet activating factor production. In this capacity, leukotrienes may be adequate for the purpose of biophylaxis; however, their overreaction can greatly increase injury to the organs.

12-LOX includes platelet 12-LOX and leukocyte 12-LOX, which oxygenates arachidonic acid at position $\mathrm{C}-12$ to produce 12-hydroperoxyeicosatetra-enoic acid, and then 12hydroxyeicosatetra-enoic acid.

Hashimoto et al reported that peroral administration of TZI-41127 (30 mg/kg) 5-LOX inhibitor (administered up to $2 \mathrm{~h}$ before ischemia and $1 \mathrm{~h}$ after reperfusion) reduced the degree of myocardial infarction at $22 \mathrm{~h}$ after reperfusion in a dog 90 min heart warm I/R injury model (14). Shishido et al reported that peroral administration of NDGA $(10 \mathrm{mg} / \mathrm{kg})$ 5-LOX inhibitor and non-selective LOX inhibitor (adminis- 
tered on 4 consecutive days and $30 \mathrm{~min}$ before ischemia) reduced the degree of brain ischemic damage at 4 days after reperfusion in a rat $5 \mathrm{~min}$ brain warm I/R injury model (15). Patel et al reported that peroral administration of zileuton (3 mg/kg) 5-LOX inhibitor reduced the degree of renal function, the expression of intercellular adhesion molecule- 1 and the associated polymorphonuclear leukocyte at $24 \mathrm{~h}$ after reperfusion in a mouse $30 \mathrm{~min}$ warm I/R injury model (16).

In conclusion, these results suggest that the suppression of COX-2 and LOX may be a potential mechanism for the reduction of $\mathrm{I} / \mathrm{R}$ injury, and that the arachidonic acid pathways play a very important role in a rat renal I/R injury model.

\section{Acknowledgements}

The authors thank Hilah Edney, B.S., M.S. for editorial assistance.

\section{References}

1. Matsuyama M, Yoshimura R, Akioka K, et al: Tissue factor antisense oligonucleotides prevent renal ischemia-reperfusion injury. Transplantation 76: 786-791, 2003.

2. Naka Y, Roy DK, Smerling AJ, et al: Inhaled nitric oxide fails to confer the pulmonary protection provided by distal stimulation of the nitric oxide pathway at the level of cyclic guanosine monophosphate. J Thorac Cardiovasc Surg 110: 1434-1440, 1995.

3. Sigal E: The molecular biology of mammalian arachidonic acid metabolism. Am J Physiol 260: 13-28, 1991.

4. Funk CD: The molecular biology of mammalian lipoxygenases and the quest for eicosanoid functions using lipoxygenasedeficient mice. Biochim Biophys Acta 11: 65-84, 1996.
5. Chang SW, Westcott JY, Pickett WC, Murphy RC and Voelkel NF: Endotoxin-induced lung injury in rats: role of eicosanoids. Appl Physiol 66: 2407-2418, 1989.

6. Brash AR: Lipoxygenases: occurrence, function, catalysis, and acquisition of substrate. J Bio Chem 274: 23679-23682, 1999.

7. Fitzgerald SM and Flinn S: Evaluating research studies using the analysis of variance (ANOVA): issues and interpretations. $\mathbf{J}$ Hand Ther 13: 56-60. 2000.

8. Simon LS: Role and regulation of cyclooxygenase-2 during inflammation. Am J Med 106: 37-42, 1999.

9. Kishimoto Y, Wada K, Nakamoto K, Kawasaki H and Hasegawa J: Levels of cyclooxygenase-1 and -2 mRNA expression at various stages of acute gastric injury induced by ischemiareperfusion in rats. Arch Biochem Biophys 352: 153-157, 1998.

10. Maricic N, Ehrlich K, Gretzer B, Schuligoi R, Respondek M and Peskar BM: Selective cyclo-oxygenase- 2 inhibitors aggravate ischaemia-reperfusion injury in the rat stomach. $\mathrm{Br} \mathrm{J}$ Pharmacol 128: 1659-1666, 1999.

11. Takamatsu Y, Shimada K, Chijiiwa K, Kuroki S, Yamaguchi K and Tanaka M: Role of leukotrienes on hepatic ischemia/reperfusion injury in rats. J Surg Res 119: 14-20, 2004.

12. Takeyoshi I, Sunose Y, Iwazaki S, et al: The effect of a selective cyclooxygenase-2 inhibitor in extended liver resection with ischemia in dogs. J Surg Res 100: 25-31, 2001.

13. Sunose Y, Takeyoshi I, Tsutsumi H, et al: Effects of FK3311 on pulmonary ischemia-reperfusion injury in a canine model. J Surg Res 95: 167-173, 2001.

14. Hashimoto H, Miyazawa K, Hagiwara M, Miyasaka K and Nakashima M: Benefical effects of a 5-lipoxygenase inhibitor on occlusion- and occlusion-reperfusion-induced myocardial injury. Arzneimittelforshung 40: 126-129, 1990.

15. Shishido Y, Furushiro M, Hashimoto S and Yokokura T: Effect of nordihydroguaiaretic acid on behavioral impairment and neuronal cell death after forebrain ischemia. Pharmacol Biochem Behav 69: 469-474, 2001.

16. Patel NS, Cuzzocrea S, Chatterjee PK, et al: Reduction of renal ischemia-reperfusion injury in 5-lipoxygenase knockout mice and by the 5-lipoxygenase inhibitor zileuton. Mol Pharmacol 66: 220-227, 2004. 